\title{
Evidence for a Common Mechanism of SIRT1 Regulation by Allosteric Activators
}

\section{Citation}

Hubbard, B. P., A. P. Gomes, H. Dai, J. Li, A. W. Case, T. Considine, T. V. Riera, et al. 2013.

"Evidence for a Common Mechanism of SIRT1 Regulation by Allosteric Activators." Science 339 (6124) (March 7): 1216-1219. doi:10.1126/science.1231097.

\section{Published Version}

doi:10.1126/science. 1231097

\section{Permanent link}

http://nrs.harvard.edu/urn-3:HUL.InstRepos:32491169

\section{Terms of Use}

This article was downloaded from Harvard University's DASH repository, and is made available under the terms and conditions applicable to Other Posted Material, as set forth at http:// nrs.harvard.edu/urn-3:HUL.InstRepos:dash.current.terms-of-use\#LAA

\section{Share Your Story}

The Harvard community has made this article openly available.

Please share how this access benefits you. Submit a story.

Accessibility 


\title{
Evidence for a Common Mechanism of SIRT1 Regulation by Allosteric Activators
}

\author{
Basil P. Hubbard ${ }^{1}$, Ana P. Gomes ${ }^{1,2}$, Han Dai ${ }^{3}$, Jun $\mathrm{Li}^{1}$, April W. Case ${ }^{3}$, Thomas \\ Considine $^{3}$, Thomas V. Riera ${ }^{3}$, Jessica E. Lee ${ }^{4}$, E Sook Yen ${ }^{4}$, Dudley W. Lamming ${ }^{1, *}$, \\ Bradley L. Pentelute ${ }^{5}$, Eli R. Schuman ${ }^{3}$, Linda A. Stevens ${ }^{6}$, Alvin J. Y. Ling ${ }^{1}$, Sean M. \\ Armour $^{1}$, Shaday Michan ${ }^{1, \dagger}$, Huizhen Zhao ${ }^{7}$, Yong Jiang ${ }^{7}$, Sharon M. Sweitzer ${ }^{7}$, Charles A. \\ Blum $^{3}$, Jeremy S. Disch ${ }^{3}$, Pui Yee Ng$^{3}$, Konrad T. Howitz ${ }^{8, \ddagger}$, Anabela P. Rolo ${ }^{2,9}$, Yoshitomo \\ Hamuro $^{4}$, Joel Moss ${ }^{6}$, Robert B. Perni ${ }^{3}$, James L. Ellis ${ }^{3}$, George P. Vlasuk ${ }^{3}$, and David A. \\ Sinclair ${ }^{1,10, \S}$ \\ ${ }^{1}$ Department of Genetics, Harvard Medical School, Boston, MA 02115, USA \\ ${ }^{2}$ Center for Neurosciences and Cell Biology, Department of Life Sciences, University of Coimbra, \\ Coimbra 3004-517, Portugal \\ ${ }^{3}$ Sirtris, a GSK Company, Cambridge, MA 02139, USA \\ ${ }^{4}$ ExSAR Corporation, Monmouth Junction, NJ 08852, USA \\ ${ }^{5}$ Department of Chemistry, Massachusetts Institute of Technology, Cambridge, MA 02139, USA \\ ${ }^{6} \mathrm{NIH}$ Cardiovascular and Pulmonary Branch/National Heart, Lung and Blood Institute, Bethesda, \\ MD 20892, USA \\ ${ }^{7}$ GlaxoSmithKline, Collegeville, PA 19426, USA \\ ${ }^{8}$ BIOMOL Research Laboratories Inc., Plymouth Meeting, PA 19462, USA \\ ${ }^{9}$ Department of Biology, University of Aveiro, Aveiro 3810-193, Portugal \\ ${ }^{10}$ Department of Pharmacology, University of New South Wales, Sydney, NSW 2052, Australia
}

\begin{abstract}
A molecule that treats multiple age-related diseases would have a major impact on global health and economics. The SIRT1 deacetylase has drawn attention in this regard as a target for drug design. Yet controversy exists around the mechanism of sirtuin-activating compounds (STACs). We found that specific hydrophobic motifs found in SIRT1 substrates such as PGC-1a and FOXO3a facilitate SIRT1 activation by STACs. A single amino acid in SIRT1, Glu ${ }^{230}$, located in a structured $\mathrm{N}$-terminal domain, was critical for activation by all previously reported STAC scaffolds and a new class of chemically distinct activators. In primary cells reconstituted with
\end{abstract}

Copyright 2013 by the American Association for the Advancement of Science; all rights reserved.

§To whom correspondence should be addressed. david_sinclair@hms.harvard.edu.

*Present address: Whitehead Institute for Biomedical Research, Cambridge, MA 02142, USA.

†Present address: Instituto Nacional de Geriatría, Institutos Nacionales de Salud, México D.F. 04510, México.

\$Present address: Reaction Biology Corporation, Malvern, PA 19355, USA.

Supplementary Materials

www.sciencemag.org/cgi/content/full/339/6124/1216/DC1

Materials and Methods

Figs. S1 to S20

Tables S1 to S8

Reference (31) 
activation-defective SIRT1, the metabolic effects of STACs were blocked. Thus, SIRT1 can be directly activated through an allosteric mechanism common to chemically diverse STACs.

The nicotinamide adenine dinucleotide $\left(\mathrm{NAD}^{+}\right)$-dependent deacetylase SIRT1 is implicated in the prevention of many age-related diseases such as cancer, Alzheimer's disease, and type 2 diabetes (1). At the cellular level, SIRT1 controls DNA repair and apoptosis, circadian clocks, inflammatory pathways, insulin secretion, and mitochondrial biogenesis $(2,3)$.

Naturally occurring STACs such as resveratrol (4) and chemically unrelated synthetic STACs activate SIRT1 in vitro by lowering its peptide Michaelis constant $\left(K_{\mathrm{M}}\right)$ and produce pharmacological changes consistent with SIRT1 activation (4-7). However, the legitimacy of STACs as direct SIRT1 activators has been widely debated. In previous studies, STACs increased SIRT1 activity toward fluorophore-tagged substrates but not toward corresponding nontagged peptides (8-11). One explanation was that STACs were binding to the fluorophore-linked substrate, which would not occur in vivo (10). Alternatively, the fluorescent groups might mimic a property of natural substrates. Given that the fluorophores used in previous studies are bulky and hydrophobic $(4,5)$, we tested whether these moieties might substitute for hydrophobic amino acids in endogenous substrates.

We used a SIRT1 activity assay whereby the reaction product nicotinamide was converted to 1-alkylthio-substituted isoindoles via the nicotinamidase PNC1 (12) and orthophthalaldehyde (OPT) (13) (fig. S1, A to E). A second assay measured the SIRT1 product $O$-acetyl adenosine diphosphate ribose (OAcADPR) by mass spectrometry (14) (fig. $\mathrm{S} 2$, A to E).

A series of STACs, including STAC-1 (SRT1460) (5) and STAC-2 [compound 22 in (15)] (fig. S3), activated SIRT1 with an aminomethylcoumarin (AMC)-tagged peptide serving as a substrate via a peptide $K_{\mathrm{M}}$-lowering mechanism, similar to the action of resveratrol (fig. S4, A to C, and tables S1 and S2) (4). The AMC moiety mediated activation only when it was directly adjacent to the acetylated Lys ${ }^{9}$ of histone 3 (H3K9) at the +1 position (16); this finding demonstrates that the fluorophore has a positional requirement (Fig. 1A and fig. S5A). The fluorophore moieties at positions +1 (4) or +6 (5) were dispensable if replaced with naturally occurring hydrophobic amino acids (15) (Fig. 1B and fig. S5B).

We then tested whether native peptide sequences might also support activation (17-24). Sequences from two SIRT1 substrates supported STAC-mediated activation: mouse peroxisome proliferator-activated receptor $\mathrm{Y}$ coactivator 1a Lys ${ }^{778}$ (PGC-1a-K778) (22) and human fork-head box O3a protein Lys ${ }^{290}$ (FOXO3a-K290) (19) (Fig. 1C). STACmediated activation was dose-dependent (Fig. 1D), and relative activation was similar between the SIRT1 assays (fig. S5C).

Isothermal titration calorimetry (ITC) did not detect binding between saturating amounts of PGC-1a peptide $(2 \mathrm{mM})$ and STAC-1 $(100 \mu \mathrm{M})$ or STAC-2 $(50 \mu \mathrm{M})$, arguing against activation driven solely by substrate enhancement (fig. S6, A and B) (15). Kinetic analysis of SIRT1 activation by STAC-2 with the FOXO3a-K290 substrate revealed that rate enhancement was mediated primarily through a lowering of peptide $K_{\mathrm{M}}$ (fig. S6C). Thus, the mechanism of activation appeared to be independent of the substrate used.

The PGC-1a-K778 peptide contains aromatic, hydrophobic amino acids at the +1 and +6 positions (relative to the acetylated lysine), as does the FOXO3a-K290 peptide at position +1 . Alanine substitution of either the Tyr at the +1 position or the Phe at the +6 position of the PGC-1a peptide reduced activation, and substitution of both abolished activation completely (Fig. 2A). Similarly, for FOXO3a, substitution of the Trp at the +1 position 
blocked activation (Fig. 2B), but substitution of several N-terminal residues did not (Fig. 2, $\mathrm{A}$ and $\mathrm{B})$. A global search of nuclear acetylated proteins, conforming to the sequences $\mathrm{X}_{6^{-}}$ $\mathrm{K}(\mathrm{Ac})-\{\mathrm{Y}, \mathrm{W}, \mathrm{F}\}-\mathrm{X}_{5}, \mathrm{X}_{6}-\mathrm{K}(\mathrm{Ac})-\mathrm{X}_{5}-\{\mathrm{Y}, \mathrm{W}, \mathrm{F}\}(16)$, and the union of the two sets, identified more than 400 sequences (fig. S7A). We tested five of these native sequences and found that three of them supported activation: metallothionein-like 5 (MTL5), peptidylprolyl isomerase A (PPIA), and eukaryotic translation initiation factor $2 \alpha$ (eIF2a) (25) (fig. S7B).

An alternative peptide sequence from FOXO3a (encompassing Lys ${ }^{242}$ ) was sequentially altered to resemble the FOXO3a-K290 sequence. Substitution of the Ser at +1 with Trp did not impart the ability to activate (fig. S7C) unless in combination with a Pro substitution at the +2 position (fig. S7C). Thus, a hydrophobic residue at the +1 position is necessary but not sufficient for activation.

These data were consistent with an allosteric mechanism of SIRT1 activation $(4,15)$. To elucidate the determinants of activation in SIRT1, we screened for SIRT1 mutant proteins lacking activation (Fig. 2C). The ability of SIRT1 to be activated by resveratrol was attenuated in one mutant that substituted a lysine for a glutamate at position 230 (E230K), whether an AMC-tagged substrate (Fig. 2D) or a natural amino acid substrate was used (fig. S8A). Substitution of $\mathrm{Glu}^{230}$ with Lys or Ala attenuated SIRT1 activation by 117 chemically diverse STACs, independent of the substrate used (Fig. 2D, Fig. 3, A to D, fig. S8, B and C, and tables S3 and S4).

$\mathrm{Glu}^{230}$ is immediately N-terminal to the catalytic core of SIRT1 and is conserved from flies to humans (fig. S8D). The E230K substitution did not impair the basal catalytic activity of SIRT1, nor did it significantly alter the maximum velocity of reaction $\left(V_{\max }\right)$, the Michaelis constant for $\mathrm{NAD}^{+}\left(K_{\mathrm{M} \mathrm{NAD}}{ }^{+}\right.$), or the $K_{\mathrm{M}}$ for several peptides (fig. S9, A to E) or the median inhibitory concentration $\left(\mathrm{IC}_{50}\right.$ ) values for several SIRT1 inhibitors (fig. S10, A to E and table S5). Secondary structural elements, thermal denaturation profiles (fig. S11, A to C and table S6), melting temperatures (fig. S12, A and B), and intracellular localization patterns (fig. S13A) of wild-type and SIRT1-E230K were also similar.

To examine the entire structure of SIRT1, we used hydrogen-deuterium exchange mass spectrometry (HDXMS). No changes in protein dynamics were detected between wild-type and SIRT1-E230K. The catalytic core domain showed slow exchange, consistent with a well-defined structure (fig. S14, A and B). The $\mathrm{N}$ and $\mathrm{C}$ termini showed fast exchange (fig. $\mathrm{S} 14 \mathrm{~A}$ ), except for a small C-terminal region around residue 650 recently implicated in the regulation of SIRT1 activity $(26,27)$ and a small rigid N-terminal region, residues 190 to 244, encompassing $\mathrm{Glu}^{230}$ (Fig. 3E and fig. S14A).

The variable median effective concentration $\left(\mathrm{EC}_{50}\right) /$ dissociation constant $\left(K_{\mathrm{d}}\right)$ ratios indicate that the majority of synthetic STACs do not interact strongly with SIRT1 and likely bind to a steady-state form such as the enzyme-substrate complex (15). SIRT1 truncations of the first 183 residues did not disrupt STAC binding, but truncations to residues 195 and 225 did, coincident with a loss of activation (table S7), whereas the E230K substitution had variable effects on STAC binding (fig. S15). Together, these data indicate that SIRT1 has a structured N-terminal domain that is required for STAC binding that encompasses Glu $^{230}$, an amino acid critical for activation across a broad class of STACs.

Resveratrol and synthetic STACs increase mitochondrial function in a SIRT1-dependent manner (28-30). However, it is unclear whether this is a direct or indirect effect of STACs on SIRT1. We therefore reconstituted primary SIRT1 knockout (KO) myoblasts (30) with wild-type mouse SIRT1 or mouse SIRT1-E222K (the murine equivalent of human SIRT1E230K) (Fig. 4A and fig. S16A). STACs increased mitochondrial mass and adenosine triphosphate (ATP) content in wild-type but not SIRT1 KO myoblasts (Fig. 4, B and C, and 
fig. S16B). In myoblasts carrying SIRT1-E222K, the effects of STACs on mitochondrial mass and ATP levels were also blocked (Fig. 4, B and C, and fig. S16B). In SIRT1 KO mouse embryonic fibroblasts (MEFs) reconstituted with SIRT1-E222K (fig. S17A), the ability of STACs to increase mitochondrial mass, ATP, and mitochondrial DNA copy number was also blocked (fig. S17, B to D, and fig. S18A). At these concentrations, there was no evidence for SIRT1-independent adenosine monophosphate (AMP)-activated protein kinase phosphorylation (fig. S19A) (30) or inhibition of phosphodiesterase isoforms (table S8). These findings argue against these pathways directly mediating the effects of STACs.

The data presented here favor a mechanism of direct "assisted allosteric activation" mediated by an N-terminal activation domain in SIRT1 (fig. S20, A and B) that is responsible for at least some of the physiological effects of STACs. Thus, allosteric activation of SIRT1 by STACs remains a viable therapeutic intervention strategy for many diseases associated with aging.

\section{Supplementary Material}

Refer to Web version on PubMed Central for supplementary material.

\section{Acknowledgments}

Supported by the Glenn Foundation for Medical Research, the Ellison Medical Foundation, the Juvenile Diabetes Research Foundation, the United Mitochondrial Disease Foundation, NIH and NIAID grants, an NSERC fellowship (B.P.H.), the Portuguese Science and Technology Foundation (A.P.G.), the Intramural Research Program, and NIH/ NHLBI (L.A.S. and J.M.). D.A.S. is a consultant and inventor on patents licensed to Sirtris, a GSK company. H.D., A.W.C., T.C., T.V.R., E.R.S., H.Z., Y.J., S.M.S., C.A.B., J.S.D., P.Y.N., R.B.P., J.L.E., and G.P.V. are employees of Sirtris, a GSK company. Dedicated to the memories of Jana Perni and Harmon Rasnow. A patent application on the PNC1-OPT sirtuin assay has been filed by Harvard Medical School with D.A.S. and B.P.H. as inventors. Patent applications related to sirtuin activators have been filed by Sirtris and Biomol. Natural Sirt1 activators will be provided upon request. Synthetic STACs are provided under a material transfer agreement from Sirtris.

\section{References and Notes}

1. Sebastian C, Satterstrom KF, Haigis MC, Mostoslavsky R. J Biol Chem. 2012; 287:42444. [PubMed: 23086954]

2. Haigis MC, Sinclair DA. Annu Rev Pathol. 2010; 5:253. [PubMed: 20078221]

3. Chalkiadaki A, Guarente L. Nat Rev Endocrinol. 2012; 8:287. [PubMed: 22249520]

4. Howitz KT, et al. Nature. 2003; 425:191. [PubMed: 12939617]

5. Milne JC, et al. Nature. 2007; 450:712. [PubMed: 18046409]

6. Baur JA, et al. Nature. 2006; 444:337. [PubMed: 17086191]

7. Minor RK, et al. Sci Rep. 2011; 1:70. [PubMed: 22355589]

8. Kaeberlein M, et al. J Biol Chem. 2005; 280:17038. [PubMed: 15684413]

9. Borra MT, Smith BC, Denu JM. J Biol Chem. 2005; 280:17187. [PubMed: 15749705]

10. Pacholec M, et al. J Biol Chem. 2010; 285:8340. [PubMed: 20061378]

11. Beher D, et al. Chem Biol Drug Des. 2009; 74:619. [PubMed: 19843076]

12. Ghislain M, Talla E, François JM. Yeast. 2002; 19:215. [PubMed: 11816029]

13. Sugawara K, Oyama F. J Biochem. 1981; 89:771. [PubMed: 7287639]

14. Sauve AA, Wolberger C, Schramm VL, Boeke JD. Annu Rev Biochem. 2006; 75:435. [PubMed: 16756498]

15. Dai H, et al. J Biol Chem. 2010; 285:32695. [PubMed: 20702418]

16. Single-letter abbreviations for amino acid residues: A, Ala; C, Cys; D, Asp; E, Glu; F, Phe; G, Gly; H, His; I, Ile; K, Lys; L, Leu; M, Met; N, Asn; P, Pro; Q, Gln; R, Arg; S, Ser; T, Thr; V, Val; W, Trp; Y, Tyr; X, any amino acid. 
17. Vaquero A, et al. Mol Cell. 2004; 16:93. [PubMed: 15469825]

18. Firestein R, et al. PLoS ONE. 2008; 3:e2020. [PubMed: 18414679]

19. Brunet A, et al. Science. 2004; 303:2011. [PubMed: 14976264]

20. Kume S, et al. J Biol Chem. 2007; 282:151. [PubMed: 17098745]

21. Lan F, Cacicedo JM, Ruderman N, Ido Y. J Biol Chem. 2008; 283:27628. [PubMed: 18687677]

22. Rodgers JT, et al. Nature. 2005; 434:113. [PubMed: 15744310]

23. Yeung F, et al. EMBO J. 2004; 23:2369. [PubMed: 15152190]

24. Das C, Lucia MS, Hansen KC, Tyler JK. Nature. 2009; 459:113. [PubMed: 19270680]

25. Ghosh HS, Reizis B, Robbins PD. Sci Rep. 2011; 1:150. [PubMed: 22355666]

26. Kang H, et al. Mol Cell. 2011; 44:203. [PubMed: 22017869]

27. Pan M, Yuan H, Brent M, Ding EC, Marmorstein R. J Biol Chem. 2012; 287:2468. [PubMed: 22157016]

28. Gerhart-Hines Z, et al. EMBO J. 2007; 26:1913. [PubMed: 17347648]

29. Bernier M, et al. J Biol Chem. 2011; 286:19270. [PubMed: 21467030]

30. Price NL, et al. Cell Metab. 2012; 15:675. [PubMed: 22560220] 
A
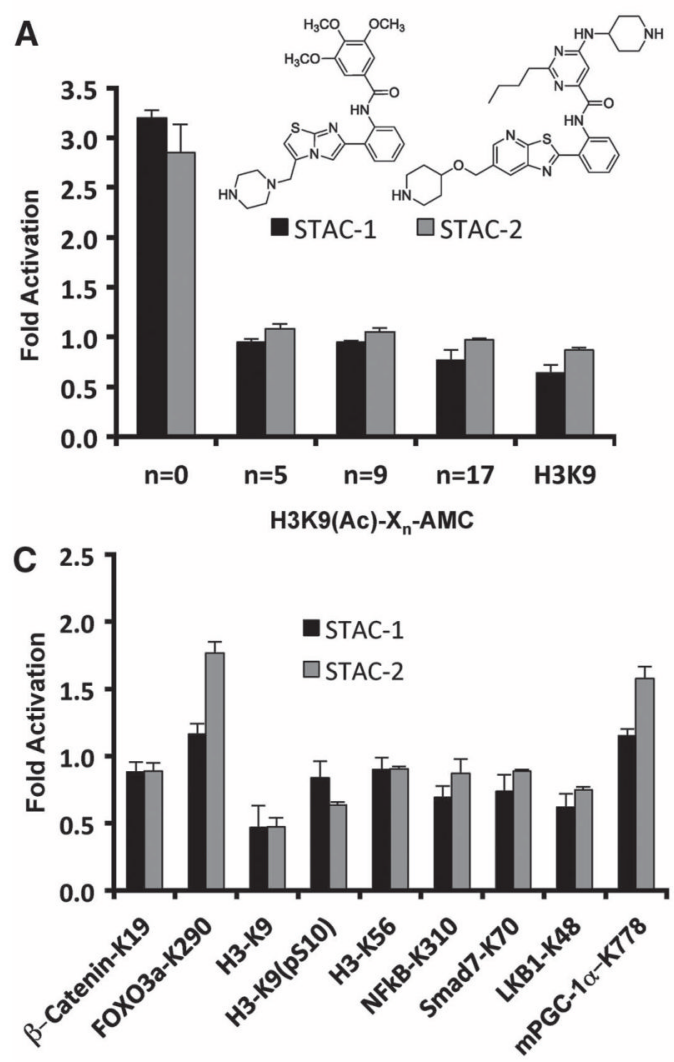

B
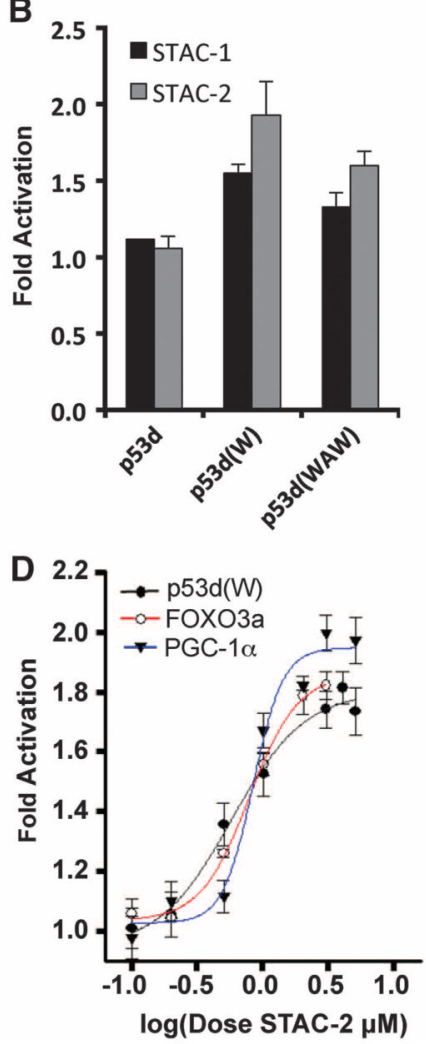

Fig. 1.

SIRT1 activation by STACs on native peptide sequences. (A) SIRT1 activation by $50 \mu \mathrm{M}$ STAC- 1 or $5 \mu \mathrm{M}$ STAC- 2 with peptides bearing an AMC moiety at the indicated positions, where $X_{n}$ represents the number of amino acids between the acetylated lysine and the AMC. (B) SIRT1 activation by STACs on hydrophobic patch peptides. Complete amino acid sequences of peptides bearing tryptophan (W) or tryptophan and alanine substitutions (WAW) are provided in the supplementary materials. (C) SIRT1 activation on native peptide sequences of known targets (detailed in the supplementary materials). (D) Doseresponse curves for STAC-2 as measured by PNC1-OPT assay; data are means \pm SEM ( $n=$ $3)$. 
A

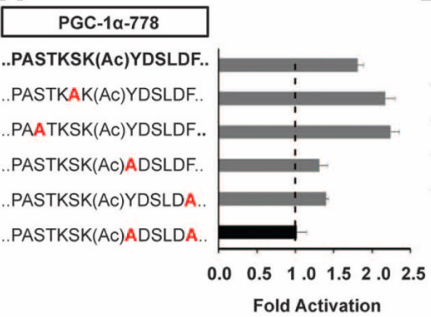

B

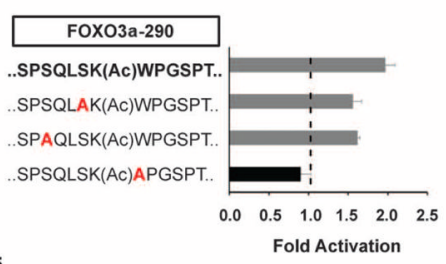

C

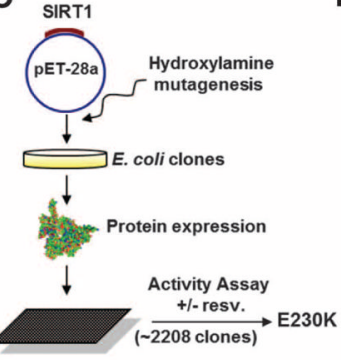

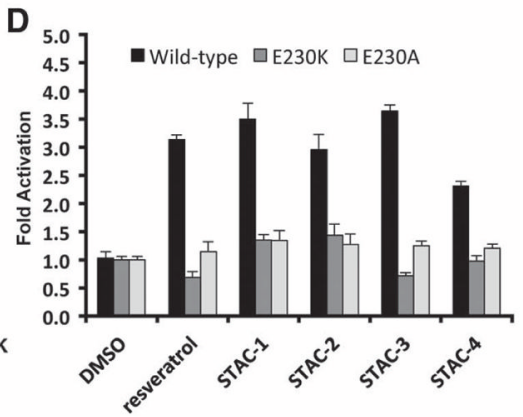

Fig. 2.

Substrate sequence requirements and regions on SIRT1 necessary for activation. (A and $\mathbf{B})$ SIRT1 activation by STAC-2 on peptides derived from PGC-1a-K778 (A) and FOXO3aK290 (B) as measured by PNC1-OPT assay; data are means \pm SEM $(n=3)$. (C)

Biochemical screen for activation-compromised mutants. A bacterial expression plasmid (pET28a) carrying the SIRT1 gene was mutagenized and used to generate recombinant SIRT1 proteins that were screened for activity in the presence or absence of resveratrol using an AMC-based assay. (D) Activation of wild-type SIRT1, E230K, and E230A mutants by $40 \mu \mathrm{M}$ resveratrol, $50 \mu \mathrm{M}$ STAC-1, $5 \mu \mathrm{M}$ STAC-2, $5 \mu \mathrm{M}$ STAC-3, and $10 \mu \mathrm{M}$ STAC-4 as measured by an AMC assay; data are means $\pm \mathrm{SD}(n=3)$. Dimethyl sulfoxide (DMSO) was used as a control. 

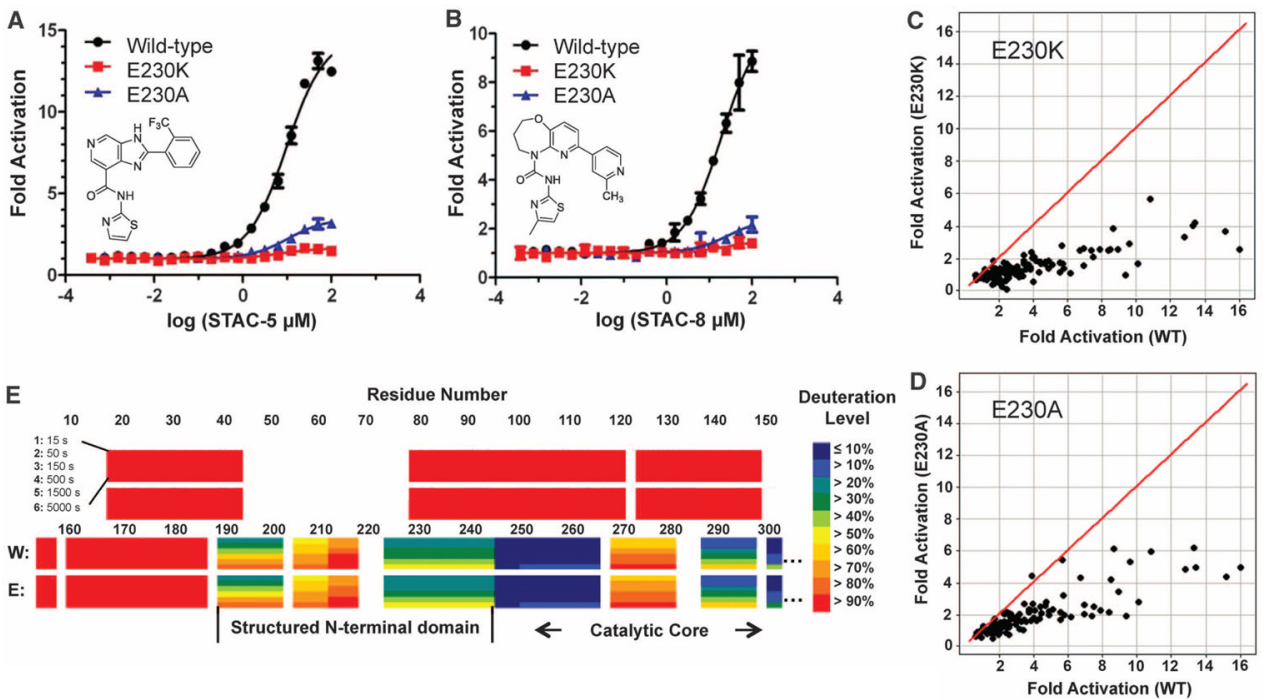

Fig. 3.

Effects of SIRT1-E230 substitutions on activation and identification of an ordered activation domain. (A and B) Dose-response titrations of STAC-5 (A) and STAC-8 (B) on the activity of wild-type SIRT1 and E230 mutants with the Trp 5-mer peptide serving as the substrate, as measured by mass spectrometry-based OAcADPR assay. The sequence of the Trp 5-mer peptide is included in the supplementary materials. data are means $\pm \operatorname{SD}(n=3)$. (C and $\mathbf{D})$ Relative activation by a chemically diverse, 117-compound set $(25 \mu \mathrm{M})$ using the Trp 5-mer substrate for wild-type versus E230K (C) or wild-type versus E230A (D), as measured by OAcADPR assay $(n=2)$. The red line represents $y=x$ correlation. (E) HDXMS heat map of deuteration levels of wild-type (W) and SIRT1-E230K (E) N termini at six different time points (15 to $5000 \mathrm{~s})$. 

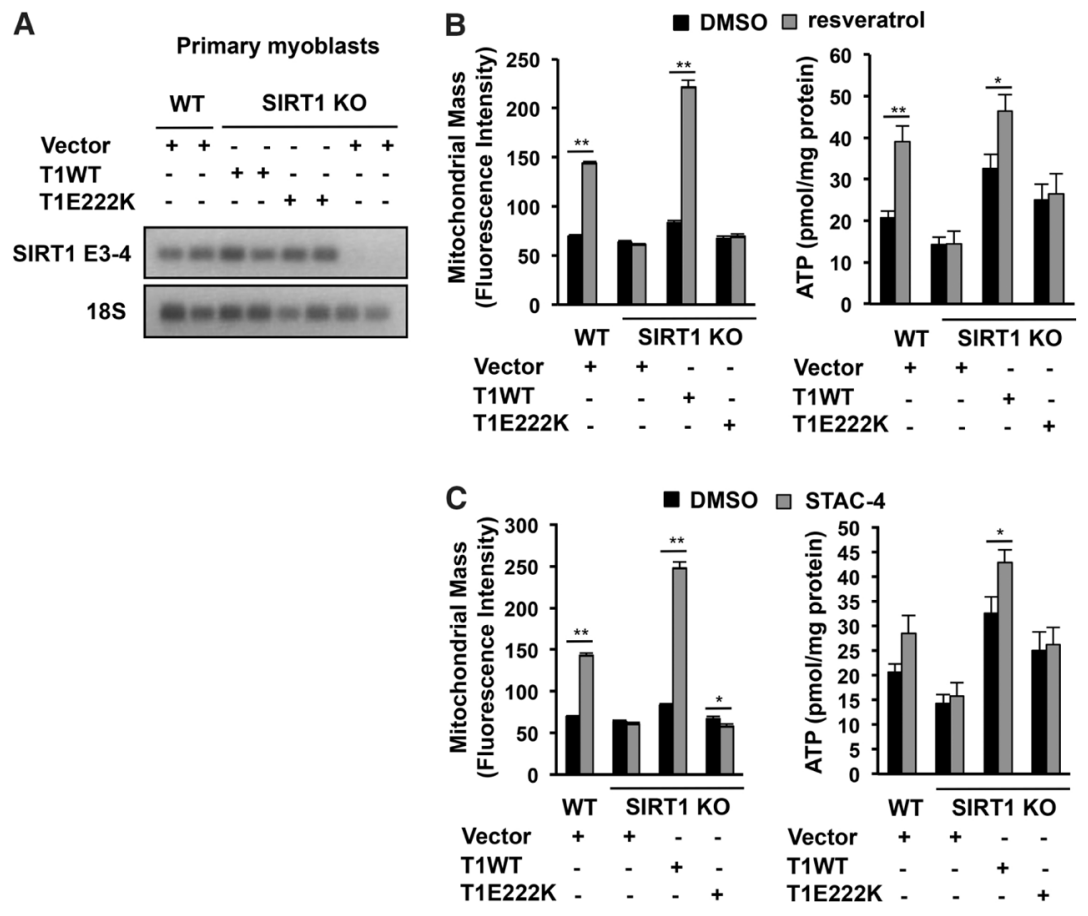

Fig. 4.

mSIRT1-E222K-dependent effects of STACs on mitochondrial-related parameters in cells. (A) Full-length murine SIRT1 (mSIRT1) transcripts in wild-type and primary myoblasts reconstituted with wild-type mSIRT1 or mSIRT1-E222K. The SIRT1 exon 3-4 junction (SIRT1 E3-4) and $18 S$ ribsosomal RNA, as an internal control for loading, were detected by reverse transcription polymerase chain reaction. (B and C) Effect of $25 \mu \mathrm{M}$ resveratrol (B) or $1 \mu \mathrm{M}$ STAC-4 $(\mathrm{C})$ on mitochondrial mass and ATP in primary myoblasts; data are means $\pm \operatorname{SEM}(n=6) . * P<0.05, * * P<0.01$ ( $t$ test versus DMSO control). 